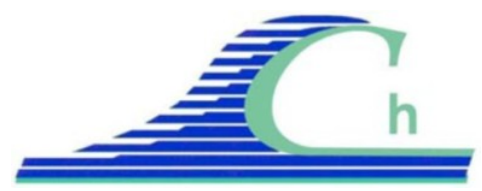

XII ${ }^{\text {ìmes }}$ Journées Nationales Génie Côtier - Génie Civil

Cherbourg, 12-14 juin 2012

DOI:10.5150/jngcgc.2012.112-K C Editions Paralia CFL

disponible en ligne - http://www.paralia.fr - available online

\title{
Valorisation des sédiments marins contaminés dans les mortiers dans le cadre de la restauration des darses du port d'Oran et la protection de l'environnement
}

\section{Fatiha KAZI AOUAL-BENSLAFA ${ }^{1}$, Mustafa HACINI ${ }^{1}$, Abdelaziz SEMCHA ${ }^{1}$, Belkacem MEKERTA ${ }^{1}$}

\author{
1. Laboratoire "LabMat", Enset Oran ; BP 1523 Oran El Ménaouer, \\ 31000 Oran, Algérie. \\ fkaziaoual@yahoo.fr ; hacinimust@yahoo.fr ; \\ asemcha@yahoo.fr; mekertab@yahoo.fr
}

\section{Résumé :}

Le travail présenté est réalisé dans le but d’intégrer la gestion des sédiments de dragage dans une démarche de développement durable. L'objectif de ce travail est d'utiliser les sédiments de dragage du port d'Oran comme composant de substitution dans la confection de matériaux de construction et plus précisément dans les mortiers. L'étude a consisté à élaborer des mortiers en substituant des fractions de sable de 10\%, 15\% et 20\%, par des sédiments de dragage. Les résistances mécaniques et aux attaques chimiques (durabilité) ont été déterminées et comparées à celles d’un mortier témoin. Les résultats obtenus en laboratoire ouvrent des perspectives de valorisation des sédiments de dragage venant en substitution du sable dans la composition des mortiers.

\section{Mots-clés :}

Valorisation - Mortier - Sédiments marins - Résistance à la compression - Durabilité Substitution - Sable

\section{Introduction}

Le port d'Oran est considéré comme le plus important port sur la façade maritime ouest de l'Algérie, mais l'envasement de ses darses représente un réel obstacle au développement de l'activité économique. Le curage de fond devient alors une opération d'entretien indispensable à la navigation et à la restauration des ouvrages portuaires. Se pose alors la question du devenir des sédiments dragués à laquelle il convient d'apporter des solutions respectueuses de l'environnement. Ce travail fait suite à l'étude réalisée en 2011 (KAZI AOUAL-BENSLAFA et al., 2011), dont l'objectif est d'étudier la faisabilité d'utiliser les sédiments de dragage comme matériaux de substitution. La filière de valorisation visée a concerné les mortiers, en substituant le sable par des sédiments de dragage. L'utilisation des sédiments de dragage en substitution d'une fraction de sable dans la composition des mortiers, a déjà fait l'objet d'études ponctuelles (BEN ALLAL et al., 2011). Les résultats obtenus par BEN ALLAL et al. (2011) montrent une diminution de l’ordre de 30\% de la résistance à la compression 
pour un mortier dont la composition a été modifiée par la substitution d'une fraction de 20\% de sable par des sédiments bruts.

Le présent travail s'inscrit dans une démarche d'aide à l'amélioration de la gestion des sédiments de dragage du port d'Oran par la proposition de filières de valorisation techniquement et économiquement viables. Ceci a nécessité la connaissance des sédiments pour l'établissement de leur fiche d’identité (LEVACHER et al., 2011). La détermination des différentes propriétés des sédiments grâce à leur caractérisation physique, chimique et minéralogique est donc nécessaire. Les sédiments étudiés proviennent du port d'Oran à proximité des quais de Safi et de Casablanca dans le bassin de Mostaganem. Ces sédiments, après avoir été lavés, ont été incorporés dans des mortiers en substitution à différentes fractions de sable (10\%, $15 \%$ et $20 \%)$. Les résistances mécaniques (résistance en compression) sur des éprouvettes de mortier ayant subi ou non des agressions chimiques (immersion dans un bain acide) ont été mesurées et comparées à celles d'un mortier témoin.

\section{Caractérisation des matériaux utilisés dans les mortiers}

\subsection{Les composants du mortier témoin}

Le mortier est un mélange de ciment, de sable et d'eau. Le ciment utilisé est un CPJ CEM II/A 42,5 MPa de la cimenterie de Sig (Ouest Algérien). Il a une surface spécifique Blaine de $3200 \mathrm{~cm}^{2} / \mathrm{g}$, une masse volumique de $3100 \mathrm{~kg} / \mathrm{m}^{3}$ et ses compositions chimique et minéralogique sont données au tableau 1. Le sable utilisé est un sable corrigé de module de finesse de 2,25. C'est un sable silico - calcaire de masse volumique de $2640 \mathrm{~kg} / \mathrm{m}^{3}$ et d'une valeur au bleu VBS de 1,28. L'eau de gâchage utilisée est l'eau potable distribuée par le réseau du service public.

Tableau 1. Composition chimique et minéralogique du ciment.

\begin{tabular}{llllllll}
\hline Composants & $\mathrm{SiO}_{2}$ & $\mathrm{CaO}$ & $\mathrm{Al}_{2} \mathrm{O}_{3}$ & $\mathrm{Fe}_{2} \mathrm{O}_{3}$ & $\mathrm{MgO}$ & $\mathrm{SO}_{3}$ & P.F \\
Teneur (\%) & 20,39 & 64,13 & 5,96 & 3,57 & 1,13 & 2,57 & 2,37 \\
\hline Phases minérales & $\mathrm{C}_{3} \mathrm{~S}^{*}$ & $\mathrm{C}_{2} \mathrm{~S}^{*}$ & $\mathrm{C}_{3} A^{*}$ & $\mathrm{C}_{4} A F^{*}$ & CaO libre & Gypse \\
Teneur (\%) & 60,04 & 12,54 & 9,25 & 11,02 & 0,35 & 4 \\
\hline
\end{tabular}

Nota : P.F. pertes au feu; * Notations cimentaires

\subsection{Caractérisation des sédiments}

Les sédiments étudiés ont été prélevés aux abords des quais de Safi (S13S) et de Casablanca (S3S) du bassin de Mostaganem, dans le port d'Oran. Ce sont des prélèvements superficiels. Les caractéristiques chimiques en éléments majeurs et mineurs des sédiments bruts ont été déterminées, les valeurs moyennes sont reportées aux tableaux 2 et 3 . Des caractéristiques chimiques complémentaires (la matière 


\section{XII ${ }^{\text {èmes }}$ Journées Nationales Génie Côtier - Génie Civil \\ Cherbourg, 12-14 juin 2012}

organique (MO), le $\mathrm{pH}$, les pertes au feu et les carbonates), et physiques ont aussi été évaluées, les valeurs sont reportées au tableau 4. Ces mêmes sédiments (S3S et S13S) sont lavés, étuvés à $(103 \pm 2)^{\circ} \mathrm{C}$ et passés au tamis de $5 \mathrm{~mm}$ afin de réaliser les analyses granulométriques par tamisage à sec suivant la norme NF P 94-056 (1996) et par sédimentométrie suivant la norme NF P 94-057 (1992). La nature granulométrique moyenne et les courbes granulométriques sont données à la Figure 1. La quantité de matière organique $(\mathrm{MO})$ contenue dans les sédiments est déterminée par calcination du sédiment brut à $450^{\circ} \mathrm{C}$, la perte de masse de l'échantillon est attribuée à cette teneur (KRIBI, 2005).

Tableau 2. Teneurs en métaux lourds et hydrocarbures totaux des sédiments bruts.

\begin{tabular}{llllllll}
\hline Composants (mg/kg) & HTC & Chrome & Zinc & Cadmium & Plomb & Mercure & Nickel \\
\hline $\begin{array}{l}\text { Teneur moyenne } \\
\text { mesurée en 2005 }\end{array} 12000$ & 76 & 391 & 0,16 & 87 & 1,64 & 342,5 \\
(LEM et al.,2005) & & & & & & & \\
\hline $\begin{array}{l}\text { Seuil règlementaire } \\
\text { recommandé par le }\end{array}$ & 300 & 250 & 500 & 3 & 250 & 1,5 & 75 \\
MATE (1999) & & & & & & & \\
\hline
\end{tabular}

Nota : MATE Ministère Algérien de l'Aménagement du Territoire et de l'Environnement ; HTC : Hydrocarbures totaux calculés.

Tableau 3. Compositions moyennes en \% des éléments chimiques des sédiments bruts

\begin{tabular}{llllllllll}
\hline Composés & $\mathrm{SiO}_{2}$ & $\mathrm{CaO}$ & $\mathrm{Al}_{2} \mathrm{O}_{3}$ & $\mathrm{Fe}_{2} \mathrm{O}_{3}$ & $\mathrm{MgO}$ & $\mathrm{CL}^{-}$ & $\mathrm{SO}_{4}$ & $\mathrm{CO}_{2}$ & $\mathrm{H}_{2} \mathrm{O}$ \\
\hline $\mathrm{S} 3 \mathrm{~S}$ & 45,0 & 25,0 & 4,1 & 0,5 & 0,3 & 1,7 & 0 & 20,0 & 5,5 \\
$\mathrm{~S} 13 \mathrm{~S}$ & 54,5 & 19,0 & 4,3 & 0,4 & 0,3 & 1,6 & 0 & 15,2 & 6,6 \\
\hline
\end{tabular}

Tableau 4. Caractéristiques physiques et chimiques des sédiments bruts

\begin{tabular}{lcccccccc}
\hline Essais & $\begin{array}{c}w \\
40^{\circ} \mathrm{C}(\%)\end{array}$ & $\begin{array}{c}w \\
105^{\circ} \mathrm{C}(\%)\end{array}$ & $\begin{array}{c}\rho_{s} \\
\left(\mathrm{~kg} / \mathrm{m}^{3}\right)\end{array}$ & $\begin{array}{c}M O \\
(\%)\end{array}$ & $p H$ & $V B S$ & $\begin{array}{c}P . F . \\
(\%)\end{array}$ & $\begin{array}{c}\text { Carbonates } \\
(\%)\end{array}$ \\
\hline S3S & 55,5 & 59,9 & 2500 & 3,1 & 9,1 & 0,6 & 25,5 & 45,4 \\
S13S & 58,9 & 62,6 & 2350 & 6,2 & 8,6 & 0,9 & 21,7 & 34,5 \\
\hline
\end{tabular}
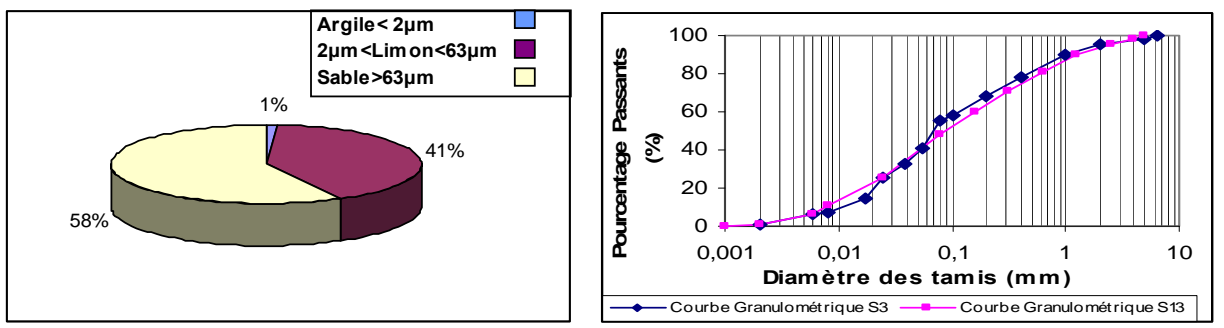

Figure 1. Nature et courbes granulométriques des sédiments (S3S et S13S). 


\subsection{Discussion et interprétation des résultats de la caractérisation des sédiments}

La comparaison des concentrations en éléments mineurs des sédiments étudiés aux niveaux de référence (MATE, 1999) révèle une contamination au nickel. Cette pollution ponctuelle est due probablement à la présence du chantier naval de Mers el Kabîr à proximité du port d’Oran. La valeur relevée en 2000 n'était que de $17 \mathrm{mg} / \mathrm{kg}$. La contamination par les hydrocarbures est élevée par rapport au niveau du seuil recommandé, ceci est probablement dû à l'activité portuaire de maintenance des bateaux. On peut conclure les sédiments sont contaminés par les hydrocarbures et le nickel. La teneur en chlorures est approximativement la même pour les deux sédiments portuaires mais cette valeur est élevée pour une utilisation des sédiments dans les matériaux à matrice cimentaire. A cet effet un traitement par lavage est préconisé. Les sédiments sont séchés au laboratoire dans une étuve à une température de $40{ }^{\circ} \mathrm{C}$, cette température correspond à la température de séchage naturel à l'air pendant la saison estivale.

Les valeurs mesurées de la masse volumique $\left(\rho_{s}\right)$ sont inférieures aux valeurs adoptées pour les matériaux locaux comme en général pour le sable $\left(2640 \mathrm{~kg} / \mathrm{m}^{3}\right)$. Cette différence est due principalement à la présence de matière organique (MO) de moindre densité (ZENTAR et al., 2009). La teneur en matière organique des sédiments du quai de Safi est égale au double de celle du quai de Casablanca, cependant les valeurs mesurées montrent que les sédiments sont faiblement organiques. Les résultats des analyses granulométriques montrent que les sédiments sont des sables limoneux, ils présentent une fraction argileuse faible $(<2 \mu \mathrm{m})$ de l'ordre de $1 \%$, une fraction limoneuse $(2 \mu \mathrm{m}$ à $63 \mu \mathrm{m})$ de l'ordre de $41 \%$ et une fraction sableuse $(>63 \mu \mathrm{m})$ de l'ordre de 58\%. L'activité de la fraction argileuse mesurée par les essais au bleu de méthylène (NF P 94-068, 1993) est faible, ce qui confirme le caractère propre des sédiments. L'analyse minéralogique est effectuée à partir des analyses qualitatives par DRX, celles-ci ont mis en évidence la présence de phases cristallines, essentiellement de quartz $\left(\mathrm{SiO}_{2}\right)$ et de calcite $\left(\mathrm{CaCO}_{3}\right)$.

Le $\mathrm{pH}$ des sédiments S3S du quai de Casablanca est plus basique que celui des sédiments S13S du quai de Safi, cette basicité peut être attribuée à la présence de carbonates (KRIBI, 2005).

\section{Valorisation des sédiments de dragage dans des matériaux à matrice cimentaire}

Les récentes études menées ont prouvé la faisabilité de l'utilisation des sédiments de dragage en substitution du sable dans la composition des matériaux de construction à matrice cimentaire (AGOSTINI et al., 2007 ; LIMEIRA et al., 2010 ; BEN ALLAL et al., 2011 ; LIMEIRA et al., 2011).

L'objectif de l'étude est de mettre en évidence l'influence de la substitution partielle du sable par les sédiments bruts lavés sur le comportement mécanique et la durabilité (perte de masse et résistance résiduelle) des mortiers. Quatre séries de mortiers ont été 


\section{XII èmes Journées Nationales Génie Côtier-Génie Civil \\ Cherbourg, 12-14 juin 2012}

préparées. Elles sont désignées par MT pour le mortier témoin, MS10, MS15, MS20, pour les mortiers contenant respectivement des pourcentages de $10 \%, 15 \%$ et $20 \%$ de sédiments bruts lavés en substitution du sable. Les mortiers sont réalisés avec un ciment algérien CPJ 42,5 dosé à $450 \mathrm{~kg} / \mathrm{m}^{3}$ pour un ratio eau sur ciment $(\mathrm{E} / \mathrm{C})$ de 0,5 . Les dosages en sable par $\mathrm{m}^{3}$ sont de $1350 \mathrm{~kg} / \mathrm{m}^{3}$ pour le mortier normal (Mortier témoin), $1215 \mathrm{~kg} / \mathrm{m}^{3}$ de sable $+135 \mathrm{~kg} / \mathrm{m}^{3}$ de sédiments pour le mortier MS10; $1147,5 \mathrm{~kg} / \mathrm{m}^{3}$ de sable $+202,5 \mathrm{~kg} / \mathrm{m}^{3}$ de sédiments pour le mortier MS15; $1080 \mathrm{~kg} / \mathrm{m}^{3}$ de sable + $270 \mathrm{~kg} / \mathrm{m}^{3}$ de sédiments pour le mortier MS20. Les éprouvettes prismatiques $\left(40 \times 40 \times 160 \mathrm{~mm}^{3}\right)$ ont été confectionnées selon la norme NF EN 196-1 (1995), puis testées. Les résistances en compression Rcj et en traction par flexion Rtfj ont été mesurées aux échéances de 7, 28, 60 et 90 jours. Les valeurs de référence sont celles obtenues pour le mortier normal sans ajout de sédiments. L'ensemble des valeurs moyennes des résistances mécaniques sont récapitulées dans le tableau 5.

Pour la résistance aux agressions chimiques, des éprouvettes cubiques $\left(50 \times 50 \times 50 \mathrm{~mm}^{3}\right)$ et des éprouvettes prismatiques $\left(40 \times 40 \times 160 \mathrm{~mm}^{3}\right)$ sont immergées dans des solutions acides avec la même concentration $3 \%$ d'acide $\mathrm{HCl}$ et $\mathrm{H}_{2} \mathrm{SO}_{4}$ après une mise en cure dans l'eau de 28 jours. Les éprouvettes cubiques $\left(50 \times 50 \times 50 \mathrm{~mm}^{3}\right)$ ont permis de mesurer la perte de masse à différentes échéances de 1 à 90 jours d'immersion. La figure 2 représente les courbes de perte de masse en fonction du temps d'immersion pour les différents mortiers soumis aux attaques des acides $\mathrm{HCl}$ (figure 2a) et $\mathrm{H}_{2} \mathrm{SO}_{4}$ (figure $2 \mathrm{~b}$ ). La résistance résiduelle $\mathrm{R}_{\mathrm{cr}}$ est mesurée sur les éprouvettes prismatiques $\left(40 \times 40 \times 160 \mathrm{~mm}^{3}\right)$ à l'échéance de 118 jours après le coulage. L'ensemble des valeurs moyennes des résistances résiduelles en compression sont récapitulées dans le tableau 6.

Tableau 5. Résistances en compression et en traction par flexion des différents mortiers.

\begin{tabular}{lllllllll}
\hline $\begin{array}{l}\text { Désignation des } \\
\text { mortiers }\end{array}$ & $\begin{array}{l}R c 7 \\
(\mathrm{MPa})\end{array}$ & $\begin{array}{l}\text { Rc28 } \\
(\mathrm{MPa})\end{array}$ & $\begin{array}{l}\text { Rc60 } \\
(\mathrm{MPa})\end{array}$ & $\begin{array}{l}\text { Rc90 } \\
(\mathrm{MPa})\end{array}$ & $\begin{array}{l}\text { Rtf7 } \\
(\mathrm{MPa})\end{array}$ & $\begin{array}{l}\text { Rtf28 } \\
(\mathrm{MPa})\end{array}$ & $\begin{array}{l}\text { Rtf60 } \\
(\mathrm{MPa})\end{array}$ & $\begin{array}{l}\text { Rtf90 } \\
(\mathrm{MPa})\end{array}$ \\
\hline Mortier Témoin MT & 31,1 & 42,1 & 43,8 & 45,3 & 4,6 & 5,6 & 6,2 & 6,9 \\
\hdashline Mortier MS10 & 28,5 & 35,8 & 38,9 & 45,5 & 3,5 & 5,1 & 5,3 & 6,1 \\
\hdashline Mortier MS15 & 30,5 & 36,9 & 39,1 & 45,9 & 3,9 & 5,1 & 5,7 & 6,3 \\
\hdashline Mortier MS20 & 30,8 & 38,5 & 41,5 & 46,6 & 4,5 & 5,3 & 6,1 & 7,5 \\
\hline
\end{tabular}

Tableau 6. Résistances Rc résiduelles des mortiers sur éprouvettes $\left(40 \times 40 \times 160 \mathrm{~mm}^{3}\right)$

\begin{tabular}{lclll}
\hline $\begin{array}{l}\text { Résistance-Rc } \\
(\mathrm{MPa})\end{array}$ & $\begin{array}{c}\text { Mortier Témoin } \\
M T\end{array}$ & Mortier MS10 & MortierMS15 & Mortier MS20 \\
\hline $\mathrm{Rc} 90$ & 45,3 & 45,5 & 45,9 & 46,6 \\
$\operatorname{Rcr}(\mathrm{HCl})$ & 11,4 & 12,9 & 13,7 & 14,6 \\
$\operatorname{Rcr}\left(\mathrm{H}_{2} \mathrm{SO}_{4}\right)$ & 20,2 & 23,4 & 24,4 & 28,8 \\
\hline
\end{tabular}



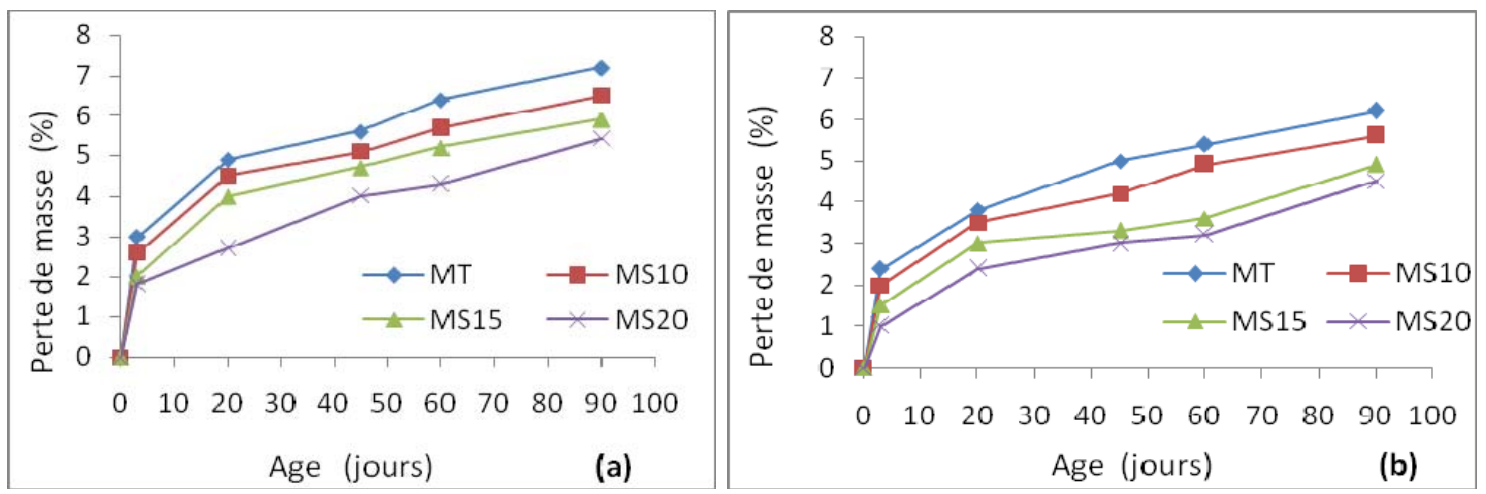

Figure 2. Perte de masse des mortiers sur éprouvettes $\left(50 \times 50 \times 50 \mathrm{~mm}^{3}\right)$ en fonction $\mathrm{du}$ temps d'immersion : (a) pour $\mathrm{HCl}$ et (b) $\mathrm{H}_{2} \mathrm{SO}_{4}$.
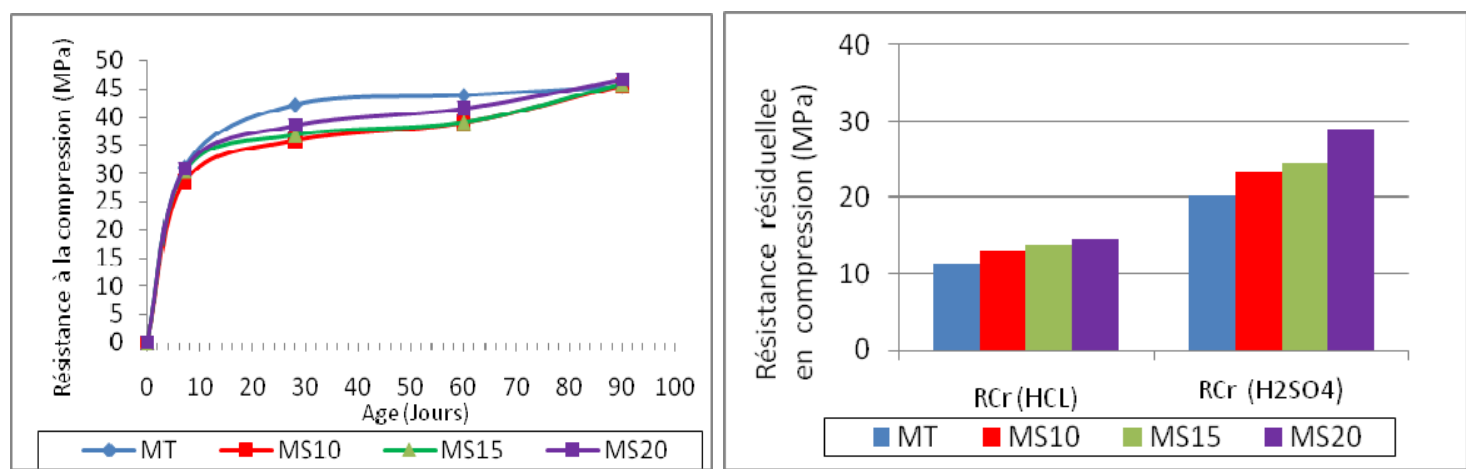

Figure 3. Evolution de la résistance à la compression (à gauche) et des résistances résiduelles en compression (à droite) des mortiers sur éprouvettes $\left(40 \times 40 \times 160 \mathrm{~mm}^{3}\right)$.

\section{Résultats et discussion}

La figure 2 représente les courbes de perte de masse en fonction du temps d'immersion pour les différents mortiers soumis aux attaques des acides $\mathrm{HCl}$ et $\mathrm{H}_{2} \mathrm{SO}_{4}$. On constate une perte de masse permanente à compter du premier jour d'immersion dans les solutions acides, celle-ci est plus importante pour l'acide $\mathrm{HCl}$ que pour l'acide $\mathrm{H}_{2} \mathrm{SO}_{4}$. Après 90 jours d'immersion dans le bain acide, la perte de masse des mortiers immergés dans l'acide $\mathrm{HCl}$, est de 7,2\%, 6,5\%, 5,9\% et 5,4\% respectivement pour le MT, MS10, MS15 et MS20. Et respectivement pour les mortiers immergés dans l'acide $\mathrm{H}_{2} \mathrm{SO}_{4}$, la perte de masse est de 6,2\%, 5,6\%, 4,9\% et 4,5\%. On remarque que les différents mortiers MS augmentent leurs aptitudes à résister aux attaques acides en fonction du pourcentage de sédiments.

L'évolution de la résistance en compression des quatre séries de mortiers est illustrée à la figure 3 (figure 3, à gauche). Elle montre clairement que le comportement général observé est conforme à celui d'un mortier et que les valeurs de la résistance en compression des mortiers MS10, MS15 et MS20 sont légèrement inférieures à celle du mortier MT jusqu'à l’âge de 60 jours. A l'échéance de 90 jours, on note que les 


\section{XII ${ }^{\text {èmes }}$ Journées Nationales Génie Côtier - Génie Civil \\ Cherbourg, 12-14 juin 2012}

résistances à la compression des mortiers MS10, MS15 sont presque égales à celle du mortier MT, même si, pour le mortier MS20 elle présente une légère augmentation de $3 \%$ par rapport à celle du mortier MT. L'évolution de la résistance résiduelle en compression des quatre séries de mortiers est donnée sur la figure 3 (figure 3, à droite). Les résistances résiduelles mesurées à 118 jours indiquent que les mortiers MS résistent mieux que le mortier MT aux attaques acides. La résistance résiduelle augmente légèrement avec le pourcentage de sédiments ajouté.

\section{Conclusions}

La caractérisation physique des sédiments de dragage du port d'Oran a révélé que ces sédiments sont des matériaux à granulométrie étalée $(0 / 5 \mathrm{~mm})$. Leurs principaux constituants sont des sables et des limons.

La caractérisation chimique des sédiments a permis d'évaluer leur potentiel polluant. On a noté une pollution métallique au nickel qui est due à l'activité industrielle dans le port ainsi qu'aux abords du port, celle-ci est probablement ponctuelle. Les sédiments de dragage du port d'Oran présente un taux élevé d'hydrocarbures.

Le taux de chlorures est assez élevé et présente un facteur limitant pour la fabrication d'un matériau à matrice cimentaire. Ces chlorures seront éliminés par lavage.

Dans le cas du mortier MS20, la résistance à la compression Rc à 90 jours obtenue est voisine de celle du mortier MT. Ce mortier MS20 augmente son aptitude à résister aux attaques acides, on note un gain dans la perte de masse par rapport à celle du mortier MT qui se traduit par une meilleure résistance résiduelle à 118 jours. Ces résistances résiduelles mesurées à 118 jours sur les autres mortiers MS10, MS15 sont légèrement supérieures à celle du mortier MT.

Dans un premier temps, on peut donc conclure que les sédiments du port d'Oran, issus des lieux de prélèvements ou à proximité, peuvent être substitués partiellement au sable utilisé dans la fabrication de matériaux à matrice cimentaire. Un pourcentage de $20 \%$ parait le plus approprié. Toutefois, l'expérimentation en phase d'application industrielle devra se poursuivre pour confirmer et valider les résultats acquis en laboratoire.

\section{Remerciements :}

Les auteurs expriment leurs sincères remerciements à M. Hamdaoui responsable du département chimie au LTPO (Laboratoire des Travaux Publiques de l'Ouest en Algérie).

\section{Références bibliographiques}

AGOSTINI F., SKOCZYLAS F., LAFHAJ Z. (2007). About a possible valorisation in cementitious materials of polluted sediments after treatment. Cement \& Concrete Composites, Vol. 29, pp 270-278. doi:10.1016/j.cemconcomp.2006.11.012 
BEN ALLAL L., AMMARI M., FRAR I., AZMANI A., BELMOKHTAR N.E. (2011). Caractérisation et valorisation des sédiments de dragage des ports de Tanger et Larache (Maroc). Revue Paralia, Vol. 4, pp 5.1-5.13. doi:10.5150/revue-paralia.2011.005 KAZI AOUAL-BENSLAFA F., MAAROUF H., SEMCHA A., MEKERTA B. (2011). Durabilité des mortiers à base de sédiments de dragage du port d'Oran. Conférence Méditerranéenne Côtière et Maritime, Tanger Maroc, pp 195-198, doi:10.5150/cmcm.2011.042.

KRIBI S. (2005). Décomposition des matières organiques et stabilisation des métaux lourds dans les sédiments de dragage. Thèse de doctorat, INSA de Lyon, 220 p.

LEM \& SONATRACH CDR -Boumerdès : Laboratoire des Etudes Maritimes et Centre de Développement de la Recherche de la SONATRACH-Boumerdès- (2005). Etude d'impact sur l'environnement du dragage des bassins du port d'Oran. Rapport, $90 \mathrm{p}$.

LEVACHER D., SANCHEZ M., DUAN Z., LIANG Y. (2011). Valorisation en unité pilote de sédiments méditerranéens : Etude des caractéristiques géotechniques et de la perméabilité, Revue Paralia, Vol. 4, pp 4.1-4.20. doi:10.5150/revue-paralia.2011.004

LIMEIRA J., ETXEBERRIA M., AGULLÓ L. (2010). Dredged marine sand in concrete: An experimental section of a harbor pavement, Construction and Building Materials, Vol. 24, pp 863-870.

LIMEIRA J., ETXEBERRIA M., AGULLÓ L., MOLINA D. (2011). Mechanical and durability properties of concrete made with dredged marine sand, Construction and Building Materials, Vol. 25, pp 4165-4174.

MATE (1999). Décret Algérien n88-228 du 05 novembre 1988 définissant les conditions, procédures et modalités d'immersion de déchets susceptibles de polluer la mer, effectuées par les navires et aéronefs. 9 p.

NF EN 196-1 AFNOR (1995). Indice de classement P15-471. Méthodes d'essais des ciments- Partie 1 : Détermination des résistances mécaniques. 27 p.

NF P 94-056 (1996). Sols: reconnaissance et essais, Analyse granulométrique des sols, Méthode par tamisage à sec.

NF P 94-057 (1992). Sols : reconnaissance et essais - Analyse granulométrique des sols - Méthode par sédimentation.

NF P 94-068 (1993). Sols : reconnaissance et essais - Mesure de la quantité et de l'activité de la fraction argileuse - Détermination de la valeur au bleu de méthylène d'un sol par l'essai à la tâche.

ZENTAR R., ABRIAK N.-E., TRAN N.T. (2009). Caractérisation et étude de l'impact sur l'environnement de sédiments dragués dans le nord de la France. Revue Paralia, $\mathrm{n}^{\circ}$ 2, pp 4.1-4.12. doi: 10.5150/revue-paralia.2009.004 